\title{
On Inequalities of Lyapunov for Two-Dimensional Nonlinear Dynamic Systems on Time Scales
}

\author{
Qiao-Luan Li, ${ }^{1}$ Wing-Sum Cheung, ${ }^{2}$ and Xu-Yang Fu ${ }^{1}$ \\ ${ }^{1}$ College of Mathematics \& Information Science, Hebei Normal University, Shijiazhuang 050024, China \\ ${ }^{2}$ Department of Mathematics, The University of Hong Kong, Hong Kong
}

Correspondence should be addressed to Wing-Sum Cheung; wscheung@hku.hk

Received 8 July 2013; Accepted 9 October 2013

Academic Editor: Allan Peterson

Copyright (C) 2013 Qiao-Luan Li et al. This is an open access article distributed under the Creative Commons Attribution License, which permits unrestricted use, distribution, and reproduction in any medium, provided the original work is properly cited.

We establish some new Lyapunov-type inequalities for two-dimensional nonlinear dynamic systems on time scales. As for application, boundedness of the Emden-Fowler-type equation is proved.

\section{Introduction}

In this paper, we establish some Lyapunov-type inequalities for the following two-dimensional nonlinear dynamic system:

$$
\begin{aligned}
x^{\Delta_{1} \Delta_{2}}(s, t)= & \alpha_{1}(s, t) x(\sigma(s), \sigma(t)) \\
& +\beta_{1}(s, t)|u(s, t)|^{\gamma-2} u(s, t), \\
u^{\Delta_{1} \Delta_{2}}(s, t)= & -\beta_{2}(s, t)|x(\sigma(s), \sigma(t))|^{\beta-2} x(\sigma(s), \sigma(t)) \\
& -\alpha_{1}(s, t) u(s, t),
\end{aligned}
$$

where $s, t \in \mathbb{T}=$ a time scale, $\Delta_{1}$ denotes the delta derivative with respect to $s$, and $\Delta_{2}$ denotes the delta derivative with respect to $t$.

Lyapunov-type inequalities have proven to be very useful in the study of qualitative behavior of solutions such as oscillation, disconjugacy, and eigenvalue problems for differential equations and difference equations. Since the appearance of Lyapunov's fundamental paper [1], considerable attention has been given to various extensions and improvements of the Lyapunov-type inequality from different viewpoints [2-8]. Although Lyapunov-type inequalities are well developed for the continuous cases, their time scale versions are still in early stages and are worth due attention.

Recently, He et al. in [2] considered the linear Hamiltonian system

$$
\begin{aligned}
& x^{\Delta}(t)=\alpha(t) x(\sigma(t))+\beta(t) y(t), \\
& y^{\Delta}(t)=-\gamma(t) x(\sigma(t))-\alpha(t) y(t)
\end{aligned}
$$

and obtained several useful Lyapunov-type inequalities.

Chen et al. in [3] considered the nonlinear system

$$
\begin{aligned}
& \frac{\partial^{2} x(s, t)}{\partial s \partial t}=\alpha_{1}(s, t) x(s, t)+\beta_{1}(s, t)|u(s, t)|^{\gamma-2} u(s, t), \\
& \frac{\partial^{2} u(s, t)}{\partial s \partial t}=-\beta_{2}(s, t)|x(s, t)|^{\beta-2} x(s, t)-\alpha_{1}(s, t) u(s, t)
\end{aligned}
$$

and obtained some interesting Lyapunov-type inequalities for partial differential equations.

In this paper, under the assumption of existence of a nontrivial solution $(x(s, t), u(s, t))$ to the 2-dimensional nonlinear dynamic system (1), some new and interesting Lyapunov-type inequalities are established. 


\section{Main Results}

Throughout this paper, the following mild and natural conditions are assumed:

(i) $\gamma>1, \beta>1$ are real constants,

(ii) $\alpha_{1}(s, t), \beta_{1}(s, t), \beta_{2}(s, t):\left[s_{0}, \infty\right)_{\mathbb{T}} \times\left[t_{0}, \infty\right)_{\mathbb{T}} \rightarrow R$ are rd-continuous functions such that $\beta_{1}(s, t)>0$ for $(s, t) \in\left[s_{0}, \infty\right)_{\mathbb{T}} \times\left[t_{0}, \infty\right)_{\mathbb{T}}$, where $\left[s_{0}, \infty\right)_{\mathbb{T}}:=$ $\left[s_{0}, \infty\right) \cap \mathbb{T}$, and $\sigma$ is the forward jump operator; that is, $\sigma(t):=\inf \{s \in \mathbb{T}: s>t\}$.

Theorem 1. If the nonlinear dynamic system (1) has a real solution $(x(s, t), u(s, t))$ which is not identically zero on $[a, b]_{\mathbb{T}} \times$ $[c, d]_{\mathbb{T}}$ satisfying $x(a, t)=x(b, t)=x(s, c)=x(s, d)=0$ and $x^{\Delta_{1}}(s, \sigma(t)) u^{\Delta_{2}}(s, t)+x^{\Delta_{2}}(\sigma(s), t) u^{\Delta_{1}}(s, t)=0$ for all $(s, t) \in$ $[a, b]_{\mathbb{V}} \times[c, d]_{\mathbb{T}}$, where $a, b, c, d \in \mathbb{T}$ with $a<b, c<d$, then

$$
\begin{aligned}
2 \leq \int_{a}^{b} \int_{c}^{d}\left|\alpha_{1}(s, t)\right| \Delta t \Delta s \\
+M^{\beta / \alpha-1}\left(\int_{a}^{b} \int_{c}^{d} \beta_{1}(s, t) \Delta t \Delta s\right)^{1 / \gamma} \\
\\
\quad\left(\int_{a}^{b} \int_{c}^{d} \beta_{2}^{+}(s, t) \Delta t \Delta s\right)^{1 / \alpha}
\end{aligned}
$$

where $1 / \alpha+1 / \gamma=1, M:=\max \{|x(s, t)|: \sigma(a) \leq s<b, \sigma(c) \leq$ $t<d\}$, and $\beta_{2}^{+}(s, t):=\max \left\{\beta_{2}(s, t), 0\right\}$.

Proof. From the conditions $x(a, t)=x(b, t)=x(s, c)=$ $x(s, d)=0$ and $x(s, t)$ is not identically zero on $[a, b]_{\mathbb{T}} \times[c, d]_{\mathbb{T}}$, there exists $\left(\tau_{1}, \tau_{2}\right)$ such that $\left|x\left(\tau_{1}, \tau_{2}\right)\right|=M>0$.

Multiplying the first equation of (1) by $u(s, t)$ and the second one by $x(\sigma(s), \sigma(t))$ and adding up, we get

$$
\begin{aligned}
& x^{\Delta_{1} \Delta_{2}}(s, t) u(s, t)+u^{\Delta_{1} \Delta_{2}}(s, t) x(\sigma(s), \sigma(t)) \\
& =\beta_{1}(s, t)|u(s, t)|^{\gamma}-\beta_{2}(s, t)|x(\sigma(s), \sigma(t))|^{\beta},
\end{aligned}
$$

and, hence,

$$
\begin{aligned}
(x(s, t) u(s, t))^{\Delta_{1} \Delta_{2}}= & x^{\Delta_{1} \Delta_{2}}(s, t) u(s, t) \\
& +x^{\Delta_{2}}(\sigma(s), t) u^{\Delta_{1}}(s, t) \\
& +x^{\Delta_{1}}(s, \sigma(t)) u^{\Delta_{2}}(s, t) \\
& +x(\sigma(s), \sigma(t)) u^{\Delta_{1} \Delta_{2}}(s, t) \\
= & x^{\Delta_{1} \Delta_{2}}(s, t) u(s, t) \\
& +u^{\Delta_{1} \Delta_{2}}(s, t) x(\sigma(s), \sigma(t)) \\
= & \beta_{1}(s, t)|u(s, t)|^{\gamma} \\
& -\beta_{2}(s, t)|x(\sigma(s), \sigma(t))|^{\beta} .
\end{aligned}
$$

Integrating the left hand side of (6) over $t$ from $c$ to $d$ and then over $s$ from $a$ to $b$, we get

$$
\begin{aligned}
\int_{a}^{b} \int_{c}^{d}(x(s, t) u(s, t))^{\Delta_{1} \Delta_{2}} \Delta t \Delta s \\
\quad=\int_{a}^{b}(x(s, d) u(s, d))^{\Delta_{1}} \Delta s-\int_{a}^{b}(x(s, c) u(s, c))^{\Delta_{1}} \Delta s \\
=x(b, d) u(b, d)-x(a, d) u(a, d) \\
\quad-x(b, c) u(b, c)+x(a, c) u(a, c) .
\end{aligned}
$$

Noting that $x(a, t)=x(b, t)=0$, we have

$$
\begin{aligned}
& \int_{a}^{b} \int_{c}^{d} \beta_{1}(s, t)|u(s, t)|^{\gamma} \Delta t \Delta s \\
& \quad=\int_{a}^{b} \int_{c}^{d} \beta_{2}(s, t)|x(\sigma(s), \sigma(t))|^{\beta} \Delta t \Delta s .
\end{aligned}
$$

On the other hand, integrating the first equation of (1) over $t$ from $c$ to $\tau_{2}$ and then over $s$ from $a$ to $\tau_{1}$, we get

$$
\begin{aligned}
\int_{a}^{\tau_{1}} \int_{c}^{\tau_{2}} x^{\Delta_{1} \Delta_{2}}(s, t) \Delta t \Delta s \\
=\int_{a}^{\tau_{1}} \int_{c}^{\tau_{2}}\left(\alpha_{1}(s, t) x(\sigma(s), \sigma(t))\right. \\
\left.\quad+\beta_{1}(s, t)|u(s, t)|^{\gamma-2} u(s, t)\right) \Delta t \Delta s .
\end{aligned}
$$

By the boundary conditions on $x$, it is elementary to verify that

$$
\int_{a}^{\tau_{1}} \int_{c}^{\tau_{2}}(x(s, t))^{\Delta_{1} \Delta_{2}} \Delta t \Delta s=x\left(\tau_{1}, \tau_{2}\right)
$$

and so

$$
\begin{aligned}
x\left(\tau_{1}, \tau_{2}\right)=\int_{a}^{\tau_{1}} \int_{c}^{\tau_{2}}( & \alpha_{1}(s, t) x(\sigma(s), \sigma(t)) \\
& \left.+\beta_{1}(s, t)|u(s, t)|^{\gamma-2} u(s, t)\right) \Delta t \Delta s .
\end{aligned}
$$

Hence,

$$
\begin{aligned}
\left|x\left(\tau_{1}, \tau_{2}\right)\right| \leq & \int_{a}^{\tau_{1}} \int_{c}^{\tau_{2}}\left|\alpha_{1}(s, t)\right||x(\sigma(s), \sigma(t))| \Delta t \Delta s \\
& +\int_{a}^{\tau_{1}} \int_{c}^{\tau_{2}} \beta_{1}(s, t)|u(s, t)|^{\gamma-1} \Delta t \Delta s .
\end{aligned}
$$

By similar arguments, we easily get

$$
\begin{aligned}
\left|x\left(\tau_{1}, \tau_{2}\right)\right| \leq & \int_{\tau_{1}}^{b} \int_{\tau_{2}}^{d}\left|\alpha_{1}(s, t)\right||x(\sigma(s), \sigma(t))| \Delta t \Delta s \\
& +\int_{\tau_{1}}^{b} \int_{\tau_{2}}^{d} \beta_{1}(s, t)|u(s, t)|^{\gamma-1} \Delta t \Delta s .
\end{aligned}
$$


Summing (12) and (13) and by Hölder's inequality with indices $\gamma$ and $\alpha$, we obtain

$$
\begin{aligned}
2\left|x\left(\tau_{1}, \tau_{2}\right)\right| \leq & \int_{a}^{b} \int_{c}^{d}\left|\alpha_{1}(s, t)\right| \\
& \times|x(\sigma(s), \sigma(t))| \Delta t \Delta s \\
& +\int_{a}^{b} \int_{c}^{d} \beta_{1}(s, t)|u(s, t)|^{\gamma-1} \Delta t \Delta s \\
\leq & \int_{a}^{b} \int_{c}^{d}\left|\alpha_{1}(s, t)\right| \\
& \times|x(\sigma(s), \sigma(t))| \Delta t \Delta s \\
& +\left(\int_{a}^{b} \int_{c}^{d} \beta_{1}(s, t) \Delta t \Delta s\right)^{1 / \gamma} \\
& \times\left(\int_{a}^{b} \int_{c}^{d} \beta_{1}(s, t)|u(s, t)|^{\gamma} \Delta t \Delta s\right)^{1 / \alpha} .
\end{aligned}
$$

In view of (8), we have

$$
\begin{aligned}
2\left|x\left(\tau_{1}, \tau_{2}\right)\right| \leq & \int_{a}^{b} \int_{c}^{d}\left|\alpha_{1}(s, t)\right||x(\sigma(s), \sigma(t))| \Delta t \Delta s \\
& +\left(\int_{a}^{b} \int_{c}^{d} \beta_{1}(s, t) \Delta t \Delta s\right)^{1 / \gamma} \\
& \times\left(\int_{a}^{b} \int_{c}^{d} \beta_{2}(s, t)|x(\sigma(s), \sigma(t))|^{\beta} \Delta t \Delta s\right)^{1 / \alpha},
\end{aligned}
$$

and so

$$
\begin{aligned}
2 \leq & \int_{a}^{b} \int_{c}^{d}\left|\alpha_{1}(s, t)\right| \Delta t \Delta s \\
& +M^{\beta / \alpha-1}\left(\int_{a}^{b} \int_{c}^{d} \beta_{1}(s, t) \Delta t \Delta s\right)^{1 / \gamma} \\
& \cdot\left(\int_{a}^{b} \int_{c}^{d} \beta_{2}^{+}(s, t) \Delta t \Delta s\right)^{1 / \alpha} .
\end{aligned}
$$

The proof is complete.

Remark 2. It is interesting to note that when $\mathbb{T}=\mathbb{R}$ Theorem 1 reduces to Theorem 2.1 of [3].

Theorem 3. If the nonlinear dynamic system (1) has a real solution $(x(s, t), u(s, t))$ which is not identically zero on $[a, b]_{\mathbb{T}} \times[c, d]_{\mathbb{T}}$ satisfying $x(s, c)=x(s, d)=$ $0, x(a, t) x(\sigma(a), t)<0, x(b, t) x(\sigma(b), t)<0$, and
$x^{\Delta_{1}}(s, \sigma(t)) u^{\Delta_{2}}(s, t)+x^{\Delta_{2}}(\sigma(s), t) u^{\Delta_{1}}(s, t)=0$ for all $(s, t) \epsilon$ $[a, b]_{\mathbb{T}} \times[c, d]_{\mathbb{T}}$, where $a, b, c, d \in \mathbb{T}$ with $a<b, c<d$, then

$$
\begin{aligned}
2 \leq & \int_{a}^{\sigma(b)} \int_{c}^{d}\left|\alpha_{1}(s, t)\right| \Delta t \Delta s \\
& +M^{\beta / \alpha-1}\left(\int_{a}^{\sigma(b)} \int_{c}^{d} \beta_{1}(s, t) \Delta t \Delta s\right)^{1 / \gamma} \\
& \cdot\left(\int_{a}^{\sigma(b)} \int_{c}^{d} \beta_{2}^{+}(s, t) \Delta t \Delta s\right)^{1 / \alpha},
\end{aligned}
$$

where $1 / \alpha+1 / \gamma=1$ and $M, \beta_{2}^{+}(s, t)$ are as defined in Theorem 1 .

Proof. Choose $\left(\tau_{1}, \tau_{2}\right)$ such that $\left|x\left(\tau_{1}, \tau_{2}\right)\right|=M$. Note that $M>0$. From (6) and

$$
\begin{aligned}
\int_{a}^{\sigma(b)} & \int_{c}^{d}(x(s, t) u(s, t))^{\Delta_{1} \Delta_{2}} \Delta t \Delta s \\
= & x(\sigma(b), d) u(\sigma(b), d)-x(a, d) u(a, d) \\
& -x(\sigma(b), c) u(\sigma(b), c)+x(a, c) u(a, c)=0
\end{aligned}
$$

we have

$$
\begin{aligned}
& \int_{a}^{\sigma(b)} \int_{c}^{d} \beta_{1}(s, t)|u(s, t)|^{\gamma} \Delta t \Delta s \\
& \quad=\int_{a}^{\sigma(b)} \int_{c}^{d} \beta_{2}(s, t)|x(\sigma(s), \sigma(t))|^{\beta} \Delta t \Delta s .
\end{aligned}
$$

By the boundary conditions on $x$, it is elementary to check that

$$
\int_{a}^{\tau_{1}} \int_{c}^{\tau_{2}}(x(s, t))^{\Delta_{1} \Delta_{2}} \Delta t \Delta s=x\left(\tau_{1}, \tau_{2}\right)-x\left(a, \tau_{2}\right) .
$$

So

$$
\begin{aligned}
x\left(\tau_{1}, \tau_{2}\right)= & x\left(a, \tau_{2}\right)+\int_{a}^{\tau_{1}} \int_{c}^{\tau_{2}} \alpha_{1}(s, t) x(\sigma(s), \sigma(t)) \Delta t \Delta s \\
& +\int_{a}^{\tau_{1}} \int_{c}^{\tau_{2}} \beta_{1}(s, t)|u(s, t)|^{\gamma-2} u(s, t) \Delta t \Delta s .
\end{aligned}
$$

For the fixed $\tau_{2}$, by $x\left(a, \tau_{2}\right) x\left(\sigma(a), \tau_{2}\right)<0$, there exists $\xi \in$ $(0,1)$ such that

$$
(1-\xi) x\left(a, \tau_{2}\right)+\xi x\left(\sigma(a), \tau_{2}\right)=0 .
$$

Integrating the first equation of (1) over $t$ from $c$ to $\tau_{2}$, we obtain

$$
\begin{aligned}
x^{\Delta_{1}} & \left(s, \tau_{2}\right)-x^{\Delta_{1}}(s, c) \\
= & \int_{c}^{\tau_{2}} \alpha_{1}(s, t) x(\sigma(s), \sigma(t)) \Delta t \\
& +\int_{c}^{\tau_{2}} \beta_{1}(s, t)|u(s, t)|^{\gamma-2} u(s, t) \Delta t .
\end{aligned}
$$


Multiplying (23) by $\mu(s)$ and noting that $x(s, c)=0$, we get

$$
\begin{aligned}
& x\left(\sigma(s), \tau_{2}\right)-x\left(s, \tau_{2}\right) \\
& =\mu(s) \int_{c}^{\tau_{2}} \alpha_{1}(s, t) x(\sigma(s), \sigma(t)) \Delta t \\
& \quad+\mu(s) \int_{c}^{\tau_{2}} \beta_{1}(s, t)|u(s, t)|^{\gamma-2} u(s, t) \Delta t .
\end{aligned}
$$

Letting $s=a$, we get

$$
\begin{aligned}
& x\left(\sigma(a), \tau_{2}\right)-x\left(a, \tau_{2}\right) \\
& =\mu(a) \int_{c}^{\tau_{2}} \alpha_{1}(a, t) x(\sigma(a), \sigma(t)) \Delta t \\
& \quad+\mu(a) \int_{c}^{\tau_{2}} \beta_{1}(a, t)|u(a, t)|^{\gamma-2} u(a, t) \Delta t .
\end{aligned}
$$

By (22) and (25), we obtain

$$
\begin{aligned}
x\left(a, \tau_{2}\right)= & -\xi \mu(a) \int_{c}^{\tau_{2}} \alpha_{1}(a, t) x(\sigma(a), \sigma(t)) \Delta t \\
& -\xi \mu(a) \int_{c}^{\tau_{2}} \beta_{1}(a, t)|u(a, t)|^{\gamma-2} u(a, t) \Delta t .
\end{aligned}
$$

Substituting (26) into (21), we get

$$
\begin{aligned}
x\left(\tau_{1}, \tau_{2}\right) & \\
= & -\xi \mu(a) \int_{c}^{\tau_{2}} \alpha_{1}(a, t) x(\sigma(a), \sigma(t)) \Delta t \\
& -\xi \mu(a) \int_{c}^{\tau_{2}} \beta_{1}(a, t)|u(a, t)|^{\gamma-2} u(a, t) \Delta t \\
& +\int_{a}^{\tau_{1}} \int_{c}^{\tau_{2}} \alpha_{1}(s, t) x(\sigma(s), \sigma(t)) \Delta t \Delta s \\
& +\int_{a}^{\tau_{1}} \int_{c}^{\tau_{2}} \beta_{1}(s, t)|u(s, t)|^{\gamma-2} u(s, t) \Delta t \Delta s \\
= & -\xi \mu(a) \int_{c}^{\tau_{2}} \alpha_{1}(a, t) x(\sigma(a), \sigma(t)) \Delta t \\
& +\mu(a) \int_{c}^{\tau_{2}} \alpha_{1}(a, t) x(\sigma(a), \sigma(t)) \Delta t \\
& -\xi \mu(a) \int_{c}^{\tau_{2}} \beta_{1}(a, t)|u(a, t)|^{\gamma-2} u(a, t) \Delta t \\
& +\mu(a) \int_{c}^{\tau_{2}} \beta_{1}(a, t)|u(a, t)|^{\gamma-2} u(a, t) \Delta t \\
& +\int_{\sigma(a)}^{\tau_{1}} \int_{c}^{\tau_{2}} \alpha_{1}(s, t) x(\sigma(s), \sigma(t)) \Delta t \Delta s
\end{aligned}
$$

$$
\begin{aligned}
& \quad+\int_{\sigma(a)}^{\tau_{1}} \int_{c}^{\tau_{2}} \beta_{1}(s, t)|u(s, t)|^{\gamma-2} u(s, t) \Delta t \Delta s \\
& =(1-\xi) \mu(a) \int_{c}^{\tau_{2}} \alpha_{1}(a, t) x(\sigma(a), \sigma(t)) \Delta t \\
& +(1-\xi) \mu(a) \int_{c}^{\tau_{2}} \beta_{1}(a, t)|u(a, t)|^{\gamma-2} u(a, t) \Delta t \\
& +\int_{\sigma(a)}^{\tau_{1}} \int_{c}^{\tau_{2}} \alpha_{1}(s, t) x(\sigma(s), \sigma(t)) \Delta t \Delta s \\
& +\int_{\sigma(a)}^{\tau_{1}} \int_{c}^{\tau_{2}} \beta_{1}(s, t)|u(s, t)|^{\gamma-2} u(s, t) \Delta t \Delta s .
\end{aligned}
$$

Immitating the arguments from (21) to (27) step by step, we have

$$
\begin{aligned}
x\left(\tau_{1}, \tau_{2}\right)= & x\left(b, \tau_{2}\right)+\int_{\tau_{1}}^{b} \int_{\tau_{2}}^{d} \alpha_{1}(s, t) x(\sigma(s), \sigma(t)) \Delta t \Delta s \\
& +\int_{\tau_{1}}^{b} \int_{\tau_{2}}^{d} \beta_{1}(s, t)|u(s, t)|^{\gamma-2} u(s, t) \Delta t \Delta s .
\end{aligned}
$$

By $x\left(b, \tau_{2}\right) x\left(\sigma(b), \tau_{2}\right)<0$, there exists $\eta \in(0,1)$ such that

$$
(1-\eta) x\left(b, \tau_{2}\right)+\eta x\left(\sigma(b), \tau_{2}\right)=0 .
$$

Integrating the first equation of (1) over $t$ from $\tau_{2}$ to $d$ and using $x(s, d)=0$, we get

$$
\begin{aligned}
x\left(b, \tau_{2}\right)= & \eta \mu(b) \int_{\tau_{2}}^{d} \alpha_{1}(b, t) x(\sigma(b), \sigma(t)) \Delta t \\
& +\eta \mu(b) \int_{\tau_{2}}^{d} \beta_{1}(b, t)|u(b, t)|^{\gamma-2} u(b, t) \Delta t
\end{aligned}
$$

and, hence,

$$
\begin{aligned}
& x\left(\tau_{1}, \tau_{2}\right) \\
& =\eta \mu(b) \int_{\tau_{2}}^{d} \alpha_{1}(b, t) x(\sigma(b), \sigma(t)) \Delta t \\
& +\eta \mu(b) \int_{\tau_{2}}^{d} \beta_{1}(b, t)|u(b, t)|^{\gamma-2} u(b, t) \Delta t \\
& +\int_{\tau_{1}}^{b} \int_{\tau_{2}}^{d} \alpha_{1}(s, t) x(\sigma(s), \sigma(t)) \Delta t \Delta s \\
& \quad+\int_{\tau_{1}}^{b} \int_{\tau_{2}}^{d} \beta_{1}(s, t)|u(s, t)|^{\gamma-2} u(s, t) \Delta t \Delta s .
\end{aligned}
$$

Let

$$
\begin{aligned}
& \tilde{\alpha}_{1}(s, t)= \begin{cases}(1-\xi) \alpha_{1}(a, t), & s=a, \\
\alpha_{1}(s, t), & \sigma(a) \leq s<b, \\
\eta \alpha_{1}(b, t), & s=b,\end{cases} \\
& \widetilde{\beta}_{1}(s, t)= \begin{cases}(1-\xi) \beta_{1}(a, t), & s=a, \\
\beta_{1}(s, t), & \sigma(a) \leq s<b, \\
\eta \beta_{1}(b, t), & s=b .\end{cases}
\end{aligned}
$$


Then, (27) can be written as

$$
\begin{aligned}
x\left(\tau_{1}, \tau_{2}\right)= & \int_{a}^{\tau_{1}} \int_{c}^{\tau_{2}} \widetilde{\alpha}_{1}(s, t) x(\sigma(s), \sigma(t)) \Delta t \Delta s \\
& +\int_{a}^{\tau_{1}} \int_{c}^{\tau_{2}} \widetilde{\beta}_{1}(s, t)|u(s, t)|^{\gamma-2} u(s, t) \Delta t \Delta s,
\end{aligned}
$$

and (31) can be written as

$$
\begin{aligned}
x\left(\tau_{1}, \tau_{2}\right)= & \int_{\tau_{1}}^{\sigma(b)} \int_{\tau_{2}}^{d} \widetilde{\alpha}_{1}(s, t) x(\sigma(s), \sigma(t)) \Delta t \Delta s \\
& +\int_{\tau_{1}}^{\sigma(b)} \int_{\tau_{2}}^{d} \widetilde{\beta}_{1}(s, t)|u(s, t)|^{\gamma-2} u(s, t) \Delta t \Delta s .
\end{aligned}
$$

It follows from (33) and (34) that

$$
\begin{aligned}
\left|x\left(\tau_{1}, \tau_{2}\right)\right| \leq & \int_{a}^{\tau_{1}} \int_{c}^{\tau_{2}}\left|\widetilde{\alpha}_{1}(s, t)\right||x(\sigma(s), \sigma(t))| \Delta t \Delta s \\
& +\int_{a}^{\tau_{1}} \int_{c}^{\tau_{2}} \widetilde{\beta}_{1}(s, t)|u(s, t)|^{\gamma-1} \Delta t \Delta s, \\
\left|x\left(\tau_{1}, \tau_{2}\right)\right| \leq & \int_{\tau_{1}}^{\sigma(b)} \int_{\tau_{2}}^{d}\left|\widetilde{\alpha}_{1}(s, t)\right||x(\sigma(s), \sigma(t))| \Delta t \Delta s \\
& +\int_{\tau_{1}}^{\sigma(b)} \int_{\tau_{2}}^{d} \widetilde{\beta}_{1}(s, t)|u(s, t)|^{\gamma-1} \Delta t \Delta s .
\end{aligned}
$$

Using (19), $\left|\widetilde{\alpha}_{1}(s, t)\right| \leq\left|\alpha_{1}(s, t)\right|, \widetilde{\beta}_{1}(s, t) \leq \beta_{1}(s, t)$, and Hölder's inequality, we have

$$
\begin{aligned}
2 \mid x( & \left.\tau_{1}, \tau_{2}\right) \mid \\
\leq & \int_{a}^{\sigma(b)} \int_{c}^{d}\left|\widetilde{\alpha}_{1}(s, t)\right||x(\sigma(s), \sigma(t))| \Delta t \Delta s \\
& +\int_{a}^{\sigma(b)} \int_{c}^{d} \widetilde{\beta}_{1}(s, t)|u(s, t)|^{\gamma-1} \Delta t \Delta s \\
\leq & \int_{a}^{\sigma(b)} \int_{c}^{d}\left|\alpha_{1}(s, t)\right||x(\sigma(s), \sigma(t))| \Delta t \Delta s \\
& +\int_{a}^{\sigma(b)} \int_{c}^{d} \beta_{1}(s, t)|u(s, t)|^{\gamma-1} \Delta t \Delta s \\
\leq & \int_{a}^{\sigma(b)} \int_{c}^{d}\left|\alpha_{1}(s, t)\right||x(\sigma(s), \sigma(t))| \Delta t \Delta s \\
& +\left(\int_{a}^{\sigma(b)} \int_{c}^{d} \beta_{1}(s, t) \Delta t \Delta s\right)^{1 / \gamma} \\
& \times\left(\int_{a}^{\sigma(b)} \int_{c}^{d} \beta_{1}(s, t)|u(s, t)|^{\gamma} \Delta t \Delta s\right)^{1 / \alpha}
\end{aligned}
$$

$$
\begin{aligned}
\leq & \int_{a}^{\sigma(b)} \int_{c}^{d}\left|\alpha_{1}(s, t)\right||x(\sigma(s), \sigma(t))| \Delta t \Delta s \\
& +\left(\int_{a}^{\sigma(b)} \int_{c}^{d} \beta_{1}(s, t) \Delta t \Delta s\right)^{1 / \gamma} \\
& \times\left(\int_{a}^{\sigma(b)} \int_{c}^{d} \beta_{2}(s, t)|x(\sigma(s), \sigma(t))|^{\beta} \Delta t \Delta s\right)^{1 / \alpha} \\
\leq & M \int_{a}^{\sigma(b)} \int_{c}^{d}\left|\alpha_{1}(s, t)\right| \Delta t \Delta s \\
& +M^{\beta / \alpha}\left(\int_{a}^{\sigma(b)} \int_{c}^{d} \beta_{1}(s, t) \Delta t \Delta s\right)^{1 / \gamma} \\
& \times\left(\int_{a}^{\sigma(b)} \int_{c}^{d} \beta_{2}^{+}(s, t) \Delta t \Delta s\right)^{1 / \alpha} .
\end{aligned}
$$

Therefore,

$$
\begin{aligned}
2 \leq \int_{a}^{\sigma(b)} & \int_{c}^{d}\left|\alpha_{1}(s, t)\right| \Delta t \Delta s \\
& +M^{\beta / \alpha-1}\left(\int_{a}^{\sigma(b)} \int_{c}^{d} \beta_{1}(s, t) \Delta t \Delta s\right)^{1 / \gamma} \\
& \cdot\left(\int_{a}^{\sigma(b)} \int_{c}^{d} \beta_{2}^{+}(s, t) \Delta t \Delta s\right)^{1 / \alpha} .
\end{aligned}
$$

The proof is complete.

Theorem 4. Suppose that $\beta$ and $\gamma$ are conjugate exponents; that is, $1 / \beta+1 / \gamma=1$. If the nonlinear dynamic system (1) has a real solution $(x(s, t), u(s, t))$ which is not identically zero on $[a, b]_{\mathbb{T}} \times[c, d]_{\mathbb{T}}$ such that $x(a, t)=x(s, c)=x(s, d)=0$ and $x^{\Delta_{1}}(s, \sigma(t)) u^{\Delta_{2}}(s, t)+x^{\Delta_{2}}(\sigma(s), t) u^{\Delta_{1}}(s, t)=0$ for all $(s, t) \in[a, b]_{\mathbb{T}} \times[c, d]_{\mathbb{T}}$, where $a, b, c, d \in \mathbb{T}$ with $a<b, c<d$, then

$$
\begin{aligned}
1 \leq & \int_{a}^{\rho(b)} \int_{c}^{d}\left|\alpha_{1}(s, t)\right| \Delta t \Delta s+\left(\int_{a}^{\rho(b)} \int_{c}^{d} \beta_{1}(s, t) \Delta t \Delta s\right)^{1 / \gamma} \\
& \cdot\left(\int_{a}^{\rho(b)} \int_{c}^{d} \beta_{2}^{+}(s, t) \Delta t \Delta s\right)^{1 / \beta} .
\end{aligned}
$$

Proof. By (6) and the conditions $x(s, c)=x(s, d)=0$ for all $s \in[a, b]_{\mathbb{T}}$, we have

$$
\begin{aligned}
& \int_{a}^{\rho(b)} \int_{c}^{d}(x(s, t) u(s, t))^{\Delta_{1} \Delta_{2}} \Delta t \Delta s \\
& =x(\rho(b), d) u(\rho(b), d)-x(a, d) u(a, d) \\
& \quad-x(\rho(b), c) u(\rho(b), c)+x(a, c) u(a, c)=0
\end{aligned}
$$


So

$$
\begin{aligned}
\int_{a}^{\rho(b)} & \int_{c}^{d} \beta_{1}(s, t)|u(s, t)|^{\gamma} \Delta t \Delta s \\
= & \int_{a}^{\rho(b)} \int_{c}^{d} \beta_{2}(s, t)|x(\sigma(s), \sigma(t))|^{\beta} \Delta t \Delta s .
\end{aligned}
$$

Fix $\left(\tau_{1}, \tau_{2}\right)$ such that $\left|x\left(\tau_{1}, \tau_{2}\right)\right|=\max \{|x(s, t)|: \sigma(a) \leq s<$ $b, \sigma(c) \leq t<d\}>0$. Integrating the first equation of (1) over $t$ from $c$ to $\tau_{2}$ and then over $s$ from $a$ to $\tau_{1}$, we get

$$
\begin{aligned}
x\left(\tau_{1}, \tau_{2}\right)= & \int_{a}^{\tau_{1}} \int_{c}^{\tau_{2}} \alpha_{1}(s, t) x(\sigma(s), \sigma(t)) \Delta t \Delta s \\
& +\int_{a}^{\tau_{1}} \int_{c}^{\tau_{2}} \beta_{1}(s, t)|u(s, t)|^{\gamma-2} u(s, t) \Delta t \Delta s,
\end{aligned}
$$

and so by Hölder's inequality with indices $\gamma$ and $\beta$ we have

$$
\begin{aligned}
\left|x\left(\tau_{1}, \tau_{2}\right)\right| \leq & \int_{a}^{\rho(b)} \int_{c}^{d}\left|\alpha_{1}(s, t)\right||x(\sigma(s), \sigma(t))| \Delta t \Delta s \\
& +\int_{a}^{\rho(b)} \int_{c}^{d} \beta_{1}(s, t)|u(s, t)|^{\gamma-1} \Delta t \Delta s \\
\leq & \int_{a}^{\rho(b)} \int_{c}^{d}\left|\alpha_{1}(s, t)\right||x(\sigma(s), \sigma(t))| \Delta t \Delta s \\
& +\left(\int_{a}^{\rho(b)} \int_{c}^{d} \beta_{1}(s, t) \Delta t \Delta s\right)^{1 / \gamma} \\
& \cdot\left(\int_{a}^{\rho(b)} \int_{c}^{d} \beta_{1}(s, t)|u(s, t)|^{\gamma} \Delta t \Delta s\right)^{1 / \beta} \\
\leq & \left|x\left(\tau_{1}, \tau_{2}\right)\right| \cdot \int_{a}^{\rho(b)} \int_{c}^{d}\left|\alpha_{1}(s, t)\right| \Delta t \Delta s \\
& +\left(\int_{a}^{\rho(b)} \int_{c}^{d} \beta_{1}(s, t) \Delta t \Delta s\right)^{1 / \gamma} \\
& \cdot\left(\int_{a}^{\rho(b)} \int_{c}^{d} \beta_{2}(s, t)|x(\sigma(s), \sigma(t))|^{\beta} \Delta t \Delta s\right)^{1 / \beta} .
\end{aligned}
$$

Hence,

$$
\begin{aligned}
1 \leq & \int_{a}^{\rho(b)} \int_{c}^{d}\left|\alpha_{1}(s, t)\right| \Delta t \Delta s \\
& +\left(\int_{a}^{\rho(b)} \int_{c}^{d} \beta_{1}(s, t) \Delta t \Delta s\right)^{1 / \gamma} \\
& \cdot\left(\int_{a}^{\rho(b)} \int_{c}^{d} \beta_{2}^{+}(s, t) \Delta t \Delta s\right)^{1 / \beta} .
\end{aligned}
$$

The proof is complete.

Theorem 5. Suppose that $\beta$ and $\gamma$ are conjugate exponents. If the nonlinear dynamic system (1) has a real solution $(x(s, t), u(s, t))$ which is not identically zero on $[a, b]_{\mathbb{T}} \times$ $[c, d]_{\mathbb{T}}$ such that $x(b, t)=x(s, c)=x(s, d)=0$ and $x^{\Delta_{1}}(s, \sigma(t)) u^{\Delta_{2}}(s, t)+x^{\Delta_{2}}(\sigma(s), t) u^{\Delta_{1}}(s, t)=0$ for all $(s, t) \epsilon$ $[a, b]_{\mathbb{T}} \times[c, d]_{\mathbb{T}}$, where $a, b, c, d \in \mathbb{T}$ with $a<b, c<d$, then

$$
\begin{aligned}
1 \leq & \int_{\sigma(a)}^{b} \int_{c}^{d}\left|\alpha_{1}(s, t)\right| \Delta t \Delta s+\left(\int_{\sigma(a)}^{b} \int_{c}^{d} \beta_{1}(s, t) \Delta t \Delta s\right)^{1 / \gamma} \\
& \cdot\left(\int_{\sigma(a)}^{b} \int_{c}^{d} \beta_{2}^{+}(s, t) \Delta t \Delta s\right)^{1 / \beta} \cdot
\end{aligned}
$$

Proof. By (6) and the assumption that $x(s, c)=x(s, d)=0$ for all $s \in[a, b]_{\mathbb{T}}$, we get

$$
\begin{aligned}
& \int_{\sigma(a)}^{b} \int_{c}^{d}(x(s, t) u(s, t))^{\Delta_{1} \Delta_{2}} \Delta t \Delta s \\
& =x(b, d) u(b, d)-x(\sigma(a), d) u(\sigma(a), d) \\
& \quad-x(b, c) u(b, c)+x(\sigma(a), c) u(\sigma(a), c)=0 .
\end{aligned}
$$

So

$$
\begin{aligned}
& \int_{\sigma(a)}^{b} \int_{c}^{d} \beta_{1}(s, t)|u(s, t)|^{\gamma} \Delta t \Delta s \\
& \quad=\int_{\sigma(a)}^{b} \int_{c}^{d} \beta_{2}(s, t)|x(\sigma(s), \sigma(t))|^{\beta} \Delta t \Delta s .
\end{aligned}
$$

Fix $\left(\tau_{1}, \tau_{2}\right)$ such that $\left|x\left(\tau_{1}, \tau_{2}\right)\right|=\max \{|x(s, t)|: \sigma(a) \leq s \leq$ $\rho(b), \sigma(t) \leq t \leq \rho(b)\}>0$. Integrating the first equation of (1) over $t$ from $\tau_{2}$ to $d$ and then over $s$ from $\tau_{1}$ to $b$, we get

$$
\begin{aligned}
x\left(\tau_{1}, \tau_{2}\right)= & \int_{\tau_{1}}^{b} \int_{\tau_{2}}^{d} \alpha_{1}(s, t) x(\sigma(s), \sigma(t)) \Delta t \Delta s \\
& +\int_{\tau_{1}}^{b} \int_{\tau_{2}}^{d} \beta_{1}(s, t)|u(s, t)|^{\gamma-2} u(s, t) \Delta t \Delta s
\end{aligned}
$$

and so by Hölder's inequality with indices $\gamma$ and $\beta$ we have

$$
\begin{aligned}
\left|x\left(\tau_{1}, \tau_{2}\right)\right| \leq & \int_{\sigma(a)}^{b} \int_{c}^{d}\left|\alpha_{1}(s, t)\right||x(\sigma(s), \sigma(t))| \Delta t \Delta s \\
& +\int_{\sigma(a)}^{b} \int_{c}^{d} \beta_{1}(s, t)|u(s, t)|^{\gamma-1} \Delta t \Delta s \\
\leq & \int_{\sigma(a)}^{b} \int_{c}^{d}\left|\alpha_{1}(s, t)\right||x(\sigma(s), \sigma(t))| \Delta t \Delta s \\
& +\left(\int_{\sigma(a)}^{b} \int_{c}^{d} \beta_{1}(s, t) \Delta t \Delta s\right)^{1 / \gamma}
\end{aligned}
$$




$$
\begin{aligned}
& \cdot\left(\int_{\sigma(a)}^{b} \int_{c}^{d} \beta_{1}(s, t)|u(s, t)|^{\gamma} \Delta t \Delta s\right)^{1 / \beta} \\
\leq & \left|x\left(\tau_{1}, \tau_{2}\right)\right| \int_{\sigma(a)}^{b} \int_{c}^{d}\left|\alpha_{1}(s, t)\right| \Delta t \Delta s \\
+ & \left(\int_{\sigma(a)}^{b} \int_{c}^{d} \beta_{1}(s, t) \Delta t \Delta s\right)^{1 / \gamma} \\
& \cdot\left(\int_{\sigma(a)}^{b} \int_{c}^{d} \beta_{2}(s, t) \mid x\left(\sigma(s),\left.\sigma(t)\right|^{\beta} \Delta t \Delta s\right)^{1 / \beta} .\right.
\end{aligned}
$$

Hence,

$$
\begin{aligned}
1 \leq & \int_{\sigma(a)}^{b} \int_{c}^{d}\left|\alpha_{1}(s, t)\right| \Delta t \Delta s \\
& +\left(\int_{\sigma(a)}^{b} \int_{c}^{d} \beta_{1}(s, t) \Delta t \Delta s\right)^{1 / \gamma} \\
& \cdot\left(\int_{\sigma(a)}^{b} \int_{c}^{d} \beta_{2}^{+}(s, t) \Delta t \Delta s\right)^{1 / \beta} .
\end{aligned}
$$

The proof is complete.

Remark 6. Analogously, we can also consider the cases (i) $x(a, t)=x(b, t)=x(s, d)=0$ and (ii) $x(a, t)=x(b, t)=$ $x(s, c)=0$. Similar results to those in Theorem 4 and Theorem 5 can easily be arrived at. The detailed proofs are omitted here.

Next, we exhibit an application of our results. Consider the following special case of (1):

$$
\begin{gathered}
x^{\Delta_{1} \Delta_{2}}(s, t)=\beta_{1}(s, t)|u(s, t)|^{\gamma-2} u(s, t), \\
u^{\Delta_{1} \Delta_{2}}(s, t)=-\beta_{2}(s, t)|x(\sigma(s), \sigma(t))|^{\beta-2} x(\sigma(s), \sigma(t)) .
\end{gathered}
$$

Definition 7. A nontrivial solution $(x(s, t), u(s, t))$ of the dynamic equation $(50)$ defined on $\left[s_{0}, \infty\right)_{\mathbb{W}} \times\left[t_{0}, \infty\right)_{\mathbb{W}}$ is said to be proper if

$$
\sup \left\{|x(s, t)|+|u(s, t)|: s \in[a, \infty)_{\mathbb{T}}, t \in[c, \infty)_{\mathbb{T}}\right\}>0
$$

for all $a \geq s_{0}, c \geq t_{0}$. A proper solution $(x(s, t), u(s, t))$ of the dynamic system (50) is called weakly oscillatory if at least one argument has a sequence of zeros diverging to $\infty$.

Theorem 8. Assume that $u(\tau, t)$ is bounded on $\left[t_{0}, \infty\right)_{\mathbb{T}}$ for a fixed $\tau, u(s, \eta)$ is bounded on $\left[s_{0}, \infty\right)_{\mathbb{T}}$ for a fixed $\eta$,

$$
\begin{aligned}
& \int_{s_{0}}^{\infty} \int_{t_{0}}^{\infty} \beta_{1}(s, t) \Delta t \Delta s<\infty, \\
& \int_{s_{0}}^{\infty} \int_{t_{0}}^{\infty}\left|\beta_{2}(s, t)\right| \Delta t \Delta s<\infty,
\end{aligned}
$$

then every weakly oscillatory proper solution of (50) is bounded on $\left[s_{0}, \infty\right)_{\mathbb{\mathbb { V }}} \times\left[t_{0}, \infty\right)_{\mathbb{T}}$.
Proof. Let $(x(s, t), u(s, t))$ be a nontrivial weakly oscillatory proper solution of $(50)$ on $\left[s_{0}, \infty\right)_{\mathbb{T}} \times\left[t_{0}, \infty\right)_{\mathbb{T}}$, and $x(s, t)$ have a sequence of zeros diverging to $\infty$. Suppose that $\lim \sup |x(s, t)|=\infty$; then, for any positive constant $M_{0}$, there exists $\left\{S_{n}, T_{n}\right\}$ such that $\left|x\left(S_{n}, T_{n}\right)\right|>M_{0}, n=1,2, \ldots$. Since $x(s, t)$ is an oscillatory solution, there exists $I:=$ $(a, b)_{\mathbb{T}} \times(c, d)_{\mathbb{T}}$ such that $x(a, t)=x(b, t)=x(s, c)=x(s, d)=$ $0,|x(s, t)|>0$ on $I$, and $\left|x\left(\tau_{1}, \tau_{2}\right)\right|=\max \{|x(s, t)|: \sigma(a) \leq$ $s<d, \sigma(c) \leq t<d\}:=M>M_{0}$. From (52), we can choose $a, c$ sufficiently large such that

$$
\begin{gathered}
\int_{a}^{\infty} \int_{c}^{\infty} \beta_{1}(s, t) \Delta t \Delta s<M^{(\alpha-\beta) /(\alpha-1)}, \\
\int_{a}^{\infty} \int_{c}^{\infty}\left|\beta_{2}(s, t)\right| \Delta t \Delta s<1 .
\end{gathered}
$$

By Theorem 1, we have

$$
\begin{gathered}
2 \leq M^{\beta / \alpha-1}\left(\int_{a}^{b} \int_{c}^{d} \beta_{1}(s, t) \Delta t \Delta s\right)^{1 / \gamma} \\
\cdot\left(\int_{a}^{b} \int_{c}^{d} \beta_{2}^{+}(s, t) \Delta t \Delta s\right)^{1 / \alpha},
\end{gathered}
$$

and so

$$
\begin{aligned}
2^{\alpha} \leq & M^{\beta-\alpha}\left(\int_{a}^{b} \int_{c}^{d} \beta_{1}(s, t) \Delta t \Delta s\right)^{\alpha-1} \\
& \cdot\left(\int_{a}^{b} \int_{c}^{d} \beta_{2}^{+}(s, t) \Delta t \Delta s\right) \\
\leq & M^{\beta-\alpha} \cdot M^{\alpha-\beta}=1,
\end{aligned}
$$

which contradicts $\alpha>1$. Hence, there exists a positive constant $K$ such that $|x(s, t)| \leq K$.

Integrating the second equation of the dynamic equation (50) over $t$ from $\tau_{2}$ to $t$ and then over $s$ from $\tau_{1}$ to $s$, respectively, we get

$$
\begin{aligned}
& \int_{\tau_{1}}^{s} \int_{\tau_{2}}^{t} u^{\Delta_{1} \Delta_{2}}(s, t) \Delta t \Delta s \\
& \quad=u(s, t)-u\left(\tau_{1}, t\right)-u\left(s, \tau_{2}\right)+u\left(\tau_{1}, \tau_{2}\right) \\
& \quad=-\int_{\tau_{1}}^{s} \int_{\tau_{2}}^{t} \beta_{2}(s, t)|x(\sigma(s), \sigma(t))|^{\beta-2} x(\sigma(s), \sigma(t)) \Delta t \Delta s .
\end{aligned}
$$

Since $u(\tau, t)$ is bounded on $\left[t_{0}, \infty\right)_{\mathbb{T}}$ for a fixed $\tau$ and $u(s, \eta)$ is bounded on $\left[s_{0}, \infty\right)_{\mathbb{T}}$ for a fixed $\eta$, we get

$$
\begin{aligned}
|u(s, t)| \leq & \left|u\left(\tau_{1}, t\right)+u\left(s, \tau_{2}\right)-u\left(\tau_{1}, \tau_{2}\right)\right| \\
& +\int_{\tau_{1}}^{s} \int_{\tau_{2}}^{t}\left|\beta_{2}(s, t)\right||x(\sigma(s), \sigma(t))|^{\beta-1} \Delta t \Delta s \\
\leq & \left|u\left(\tau_{1}, t\right)+u\left(s, \tau_{2}\right)-u\left(\tau_{1}, \tau_{2}\right)\right| \\
& +K^{\beta-1} \int_{\tau_{1}}^{\infty} \int_{\tau_{2}}^{\infty}\left|\beta_{2}(s, t)\right| \Delta t \Delta s .
\end{aligned}
$$


Since $\int_{s_{0}}^{\infty} \int_{t_{0}}^{\infty}\left|\beta_{2}(s, t)\right| \Delta t \Delta s<\infty,|u(s, t)|$ is bounded. So $\lim \sup \{|x(s, t)|+u(s, t) \mid\}$ is bounded on $\left[s_{0}, \infty\right)_{\mathbb{T}} \times\left[t_{0}, \infty\right)_{\mathbb{T}}$. The proof is complete.

\section{Acknowledgments}

The first author's research was supported by NNSF of China (11071054) and Natural Science Foundation of Hebei Province (A2011205012). The corresponding author's research was partially supported by an HKU URG Grant.

\section{References}

[1] A. M. Lyapunov, "Problém général de la stabilité du mouvement," Annales de la Faculté des Sciences de l'Université de Toulouse, vol. 9, no. 2, pp. 203-474, 1907.

[2] X. He, Q.-M. Zhang, and X. Tang, "On inequalities of Lyapunov for linear Hamiltonian systems on time scales," Journal of Mathematical Analysis and Applications, vol. 381, no. 2, pp. 695705, 2011.

[3] L.-Y. Chen, C.-J. Zhao, and W.-S. Cheung, "On Lyapunov-type inequalities for two-dimensional nonlinear partial systems," Journal of Inequalities and Applications, vol. 2010, Article ID 504982, 12 pages, 2010.

[4] X. Wang, "Lyapunov type inequalities for second-order halflinear differential equations," Journal of Mathematical Analysis and Applications, vol. 382, no. 2, pp. 792-801, 2011.

[5] M. Bohner, S. Clark, and J. Ridenhour, "Lyapunov inequalities for time scales," Journal of Inequalities and Applications, vol. 7, no. 1, pp. 61-77, 2002.

[6] X.-H. Tang and M. Zhang, "Lyapunov inequalities and stability for linear Hamiltonian systems," Journal of Differential Equations, vol. 252, no. 1, pp. 358-381, 2012.

[7] A. Cañada, J. A. Montero, and S. Villegas, "Lyapunov inequalities for partial differential equations," Journal of Functional Analysis, vol. 237, no. 1, pp. 176-193, 2006.

[8] X. Yang, "On inequalities of Lyapunov type," Applied Mathematics and Computation, vol. 134, no. 2-3, pp. 293-300, 2003. 


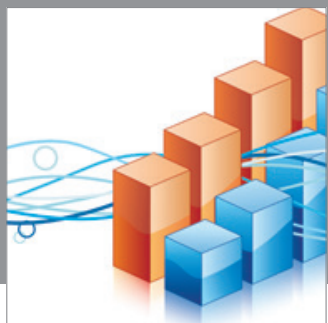

Advances in

Operations Research

mansans

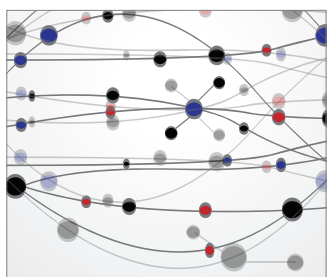

The Scientific World Journal
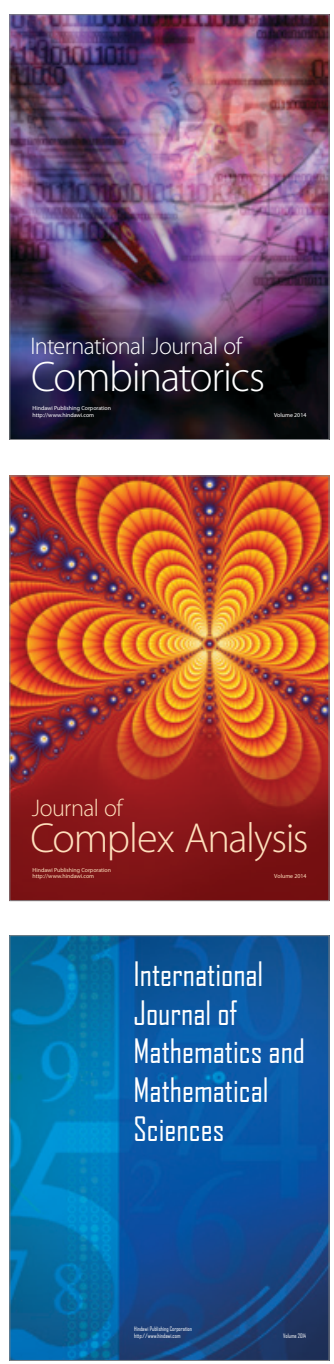
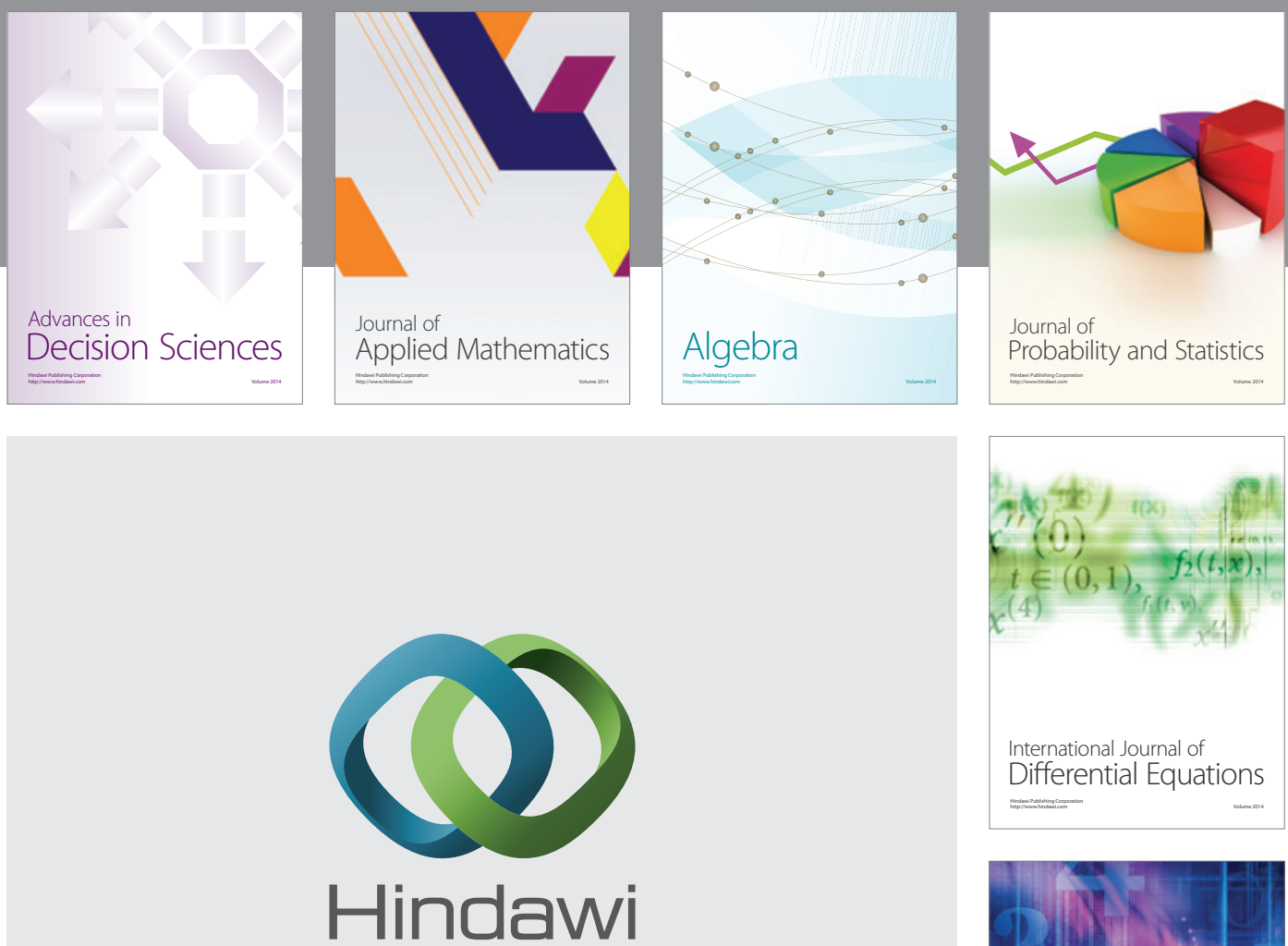

Submit your manuscripts at http://www.hindawi.com
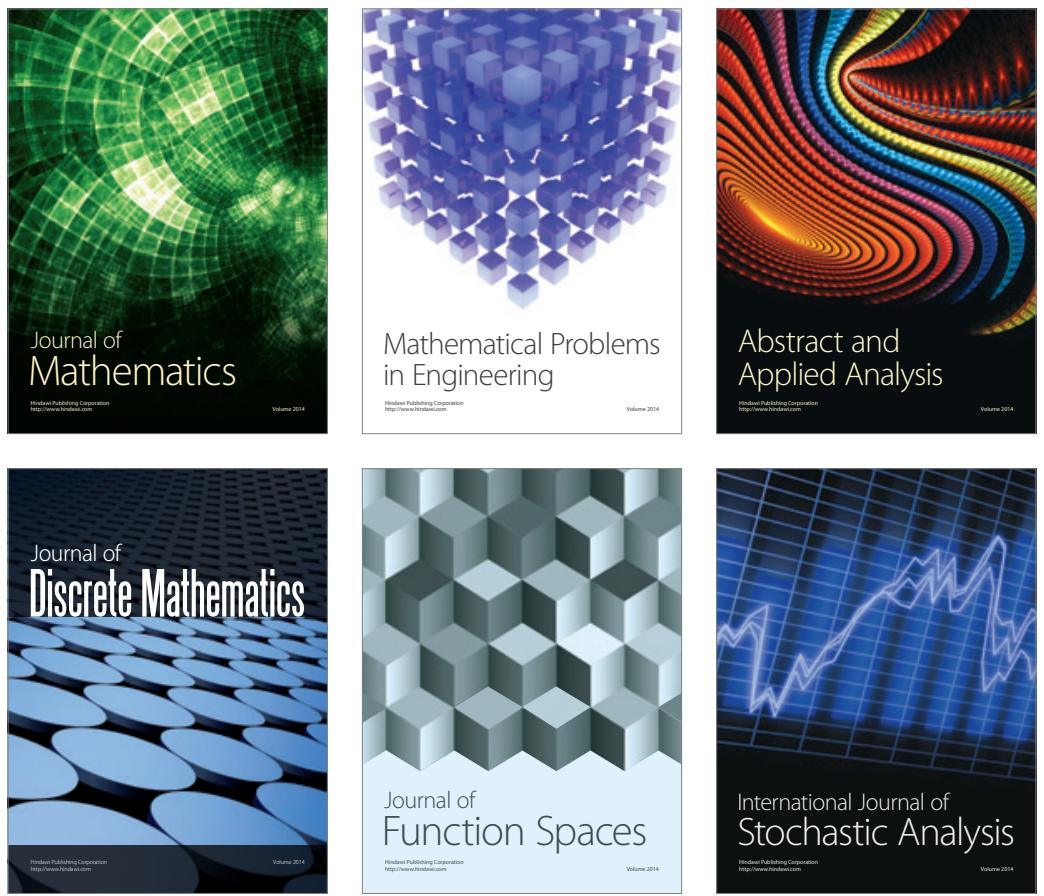

Journal of

Function Spaces

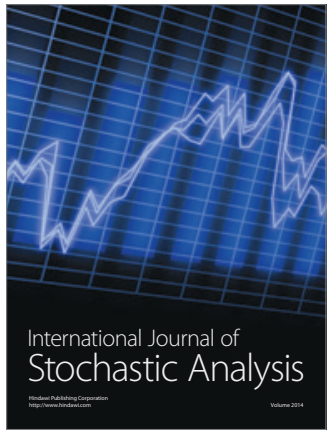

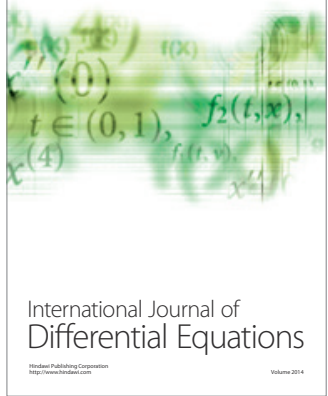
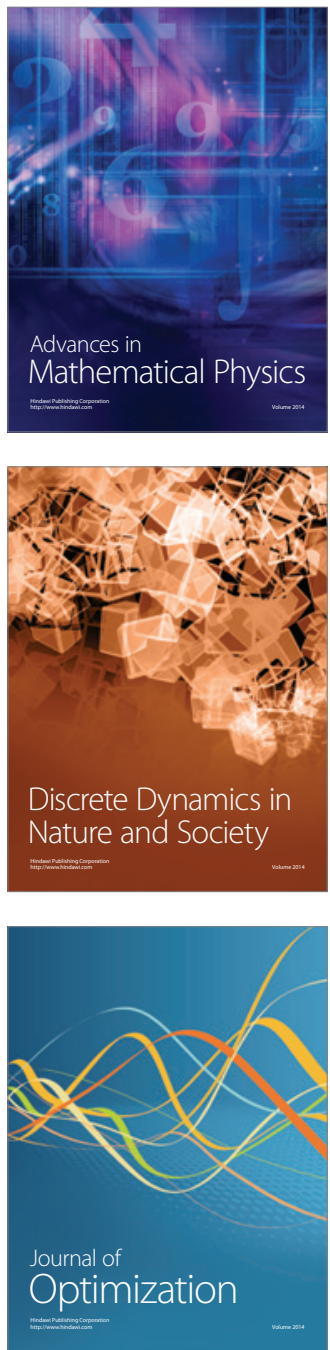